\title{
Intensiv- und Beatmungsmedizin in der Pneumologie 2009
}

\author{
Respiratory and Intensive Care Medicine in „Pneumologie“ 2009
}

Bibliografie

DOI $10.1055 / \mathrm{s}-0028-1119681$

Pneumologie 2009; 63:

193-194 @ Georg Thieme

Verlag KG Stuttgart · New York

ISSN 0934-8387

Korrespondenzadresse

Prof. Dr. Bernd Schönhofer

Abt. für Pneumologie und

Internistische Intensivmedizin

Krankenhaus Oststadt-Heidehaus

Podbielskistr. 380

30659 Hannover

Bernd.Schoenhofer@t-online.de
Die Sektion 5 „Intensiv- und Beatmungsmedizin“ der DGP hat in der letzten Zeit wichtige Projekte in der Beatmungsmedizin initiiert und erfolgreich durchgeführt, wie z.B. die S3-Leitlinie „Nicht-invasive Beatmung zur Therapie der akut respiratorischen Insuffizienz“, die Curricula „Beatmungsmedizin“ und die Akkreditierung der Weaningzentren.

Wie jedoch dem Namen unserer Sektion „Intensiv- und Beatmungsmedizin“ zu entnehmen ist, kann Beatmungsmedizin nicht isoliert betrachtet werden, sie ist gleichzeitig integraler Bestandteil der modernen Intensivmedizin. Unser Ziel ist es, dass Fachärzte und Fachärztinnen für Pneumologie auf den Intensivstationen kompetente Ansprechpartner sind und hier zunehmend Verantwortung übernehmen.

Nachdem in jüngerer Vergangenheit bereits zwei sehr gute Serien zur „Intensiv- und Beatmungsmedizin" in der Pneumologie veröffentlicht wurden, setzen wir nun diese Tradition mit der 3. Themenserie fort.

In dieser Serie werden neben zwei intensivmedizinischen Themen vier beatmungsmedizinische Themen veröffentlicht.

Zunächst werden in einem Beitrag von Joachim Lorenz aus Lüdenscheid Behandlungsstrategien in der Sepsis erläutert. Hier wird neben Konzepten zur Kontrolle der zugrundeliegenden Infektion die Behandlung hämodynamischer, respiratorischer, endokrinologischer, koagulatorischer und immunologischer Aspekte der schweren Sepsis erläutert.

In dem Artikel von Hartwig Schütte und Norbert Suttorp aus Berlin wird das neue Forschungsvorhaben PROGRESS (Pneumonia Research Network on Genetic Resistance and Susceptibility for the Evolution of Severe Sepsis) vorgestellt. Da das Überleben einer schweren Pneumonie nicht nur von den Erregern, der Erreger-Resistenzsituation oder vorbestehenden Komorbiditäten und Umwelteinflüssen abhängt, sondern auch genetische
Faktoren die Abwehrlage eines Patienten entscheidend mitbestimmen, haben sich die BMBFKompetenznetzwerke „Ambulant erworbene Pneumonie (CAPNETZ) und Sepsis (SepNet) zusammen mit dem Nationalen Genomforschungsnetz (NGFN) zum Ziel gesetzt, typische genetische Fingerabdrücke zu identifizieren, die mit einem schweren Verlauf der ambulant erworbenen Pneumonie bis hin zur Sepsis assoziiert sind. Die Ergebnisse sollen dabei helfen, die Versorgung von Patienten mit Pneumonie auf der Basis einer verbesserten Entscheidungsfindung zu optimieren.

In seinem Beitrag zu unterschiedlichen Tracheotomietechniken erläutert Stefan Kluge aus Hamburg die Möglichkeiten und Grenzen der inzwischen weit verbreiteten Punktionstracheotomie. In seinem Artikel geht Michael Pfeifer aus Regensburg auf den Stellenwert des PEEP bei der invasiven Beatmung ein. Aktuelle Studien zur Beatmungsstrategie beim akuten Lungenversagen unterstreichen positive PEEP-Effekte im Hinblick auf die Vermeidung von Alveolarkollaps, gleichzeitig kann ein zu hoher PEEP zur Überblähung gesunder Lungenanteile beitragen. Die Bedeutung des individuell zu ermittelnden PEEP und möglicher Methoden wird erläutert.

Insbesondere bei immunkompromittierten Patienten ist es im Falle eines akuten Lungenversagens essenziell, die zugrundeliegende Ursache zu klären, um eine wirksame Therapie einzuleiten. Hans Jörg Baumann aus Hamburg zeigt in seinem Artikel auf, dass eine offene Lungenbiopsie bei Patienten mit unklarem Lungenversagen und nicht richtungsweisenden klinischen, labordiagnostischen radiologischen und bronchoskopischen Befunden trotz hohem Risiko durchaus sinnvoll sein kann.

Langzeitbeatmung und schwieriges Weaning sind Schwerpunkt vieler pneumologischer Zentren und praktisch orientierte wissenschaftliche $\mathrm{Ar}$ beiten sehr hilfreich zum besseren Verständnis 
der Pathophysiologie und zur Verbesserung der Therapiekonzepte. In diesem Sinne behandelt der Artikel von Steffen Weber-Carstens aus Berlin mit der Critical Illness Polyneuromyopathie einen wichtigen Aspekt im Weaningprozess, da bis zu 50\% der langzeitbeatmeten Patienten unter muskulärer Schwäche der peripheren Muskulatur inklusive des Zwerchfelles leiden.

Der Artikel von Joachim Meyer aus Heidelberg und Bernd Schönhofer aus Hannover erläutert die kardiale Funktion bzw. Dysfunktion im Weaning. Infolge einer häufig unerkannten Herzinsuffizienz verzögert sich der Weaningprozess; nicht selten kommt es wegen einer kardialen Insuffizienz auch zum Weaningversagen.
Alle Kapitel werden von ausgewiesenen Experten verfasst, die vor Ort auf Intensivstationen tätig sind und neben wissenschaftlichen Aspekten ihre praktische Erfahrung in die Artikel einbringen. Wir halten diesen Aspekt für besonders wichtig, denn gerade in der Intensivmedizin macht die Gewichtung der Literaturauswahl durch die persönliche Erfahrung des Autoren die Qualität und praktische Relevanz einer Übersichtsarbeit aus. Wir hoffen auf ein reges Interesse der Leserschaft der Pneumologie.

Bernd Schönhofer

Simone Rosseau

Sprecher der Sektion 5 